\title{
A NONCOMMUTATIVE GAUSS MAP
}

\author{
CALEB ECKHARDT
}

\begin{abstract}
The aim of this paper is to transfer the Gauss map, which is a Bernoulli shift for continued fractions, to the noncommutative setting. We feel that a natural place for such a map to act is on the AF algebra $\mathfrak{U}$ considered separately by $\mathrm{F}$. Boca and D. Mundici. The center of $\mathfrak{U}$ is isomorphic to $C[0,1]$, so we first consider the action of the Gauss map on $C[0,1]$ and then extend the map to $\mathfrak{A}$ and show that the extension inherits many desirable properties.
\end{abstract}

\section{Introduction and Notation}

Florin Boca in [1] and Daniele Mundici in [6] separately considered an AF algebra $\mathfrak{O}$ that is associated with the Farey tessellation. The algebra $\mathfrak{A}$ exhibits many interesting properties, not the least of which is the connection between $\mathfrak{U}$ and the unit interval $[0,1]$. This connection is not merely topological, but also number theoretic. We briefly explain this connection.

Let $Z(\mathfrak{H})$ denote the center of $\mathfrak{U}$. As noted in [1], a general result of Bratteli [2] shows that $C[0,1] \cong Z(\mathfrak{V})$. Moreover, the maximal ideal space of $\mathfrak{U}$ is homeomorphic (when equipped with the topology induced by Prim( $(\mathfrak{H})$ ) to $[0,1]$ in a natural way [1, Corollary 12]. For each irrational $0<\theta<1$, let $I_{\theta}$ denote the maximal ideal of $\mathfrak{A}$ associated to $\theta$. It was shown in [1], [6] that $\mathfrak{X} / I_{\theta} \cong \mathfrak{F}_{\theta}$, the Effros-Shen algebra, defined in [4], associated with the continued fraction expansion of $\theta$.

In other words, if we employ the topological decomposition theory of $C^{*}$ algebras and visualize $\mathfrak{A}$ as continuous, operator-valued functions on its maximal ideal space (a visualization which is usually "incorrect, but fruitful" [8, pg. 91]), then each function evaluated at $\theta$ takes values in the Effros-Shen algebra $\widetilde{\mathfrak{F}}_{\theta}$. So it is not simply the topology around $\theta$ that determines this visualization, but also the continued fraction expansion of $\theta$.

Given the close connection between $\mathfrak{A}$ and the continued fraction expansions of numbers in $[0,1]$, it is natural to try and extend important functions from number theory (especially those related to continued fractions) to the $C^{*}$-algebra $\mathfrak{A}$. The Gauss map might be the most fundamental such function, hence we take it as our starting point

Received 7 July 2009. 
Recall the Gauss map $G:[0,1] \rightarrow[0,1]$ defined by $G(0)=0$ and $G(x)=1 / x-\lfloor 1 / x\rfloor$ if $x \neq 0$, where $\lfloor\cdot\rfloor$ denotes the greatest integer function. One can think of $G$ as the Bernoulli shift for continued fractions. Indeed, given $\theta \in[0,1]$ with continued fraction expansion $\theta=\left[a_{1}, a_{2}, \ldots\right]$ then $G\left(\left[a_{1}, a_{2}, \ldots\right]\right)=\left[a_{2}, a_{3}, \ldots\right]$. One can also recover the continued fraction expansion of $\theta$ by implementations of $G$ and $\lfloor\cdot\rfloor$.

We first consider the induced action of $G$ on $Z(\mathfrak{H})=C[0,1]$. First note that $f \circ G \in C[0,1]$ if and only if $f$ is a constant function. Therefore we will consider the "adjoint" action of the Gauss map on $C[0,1]$. Let $\mu$ denote Gauss measure on $[0,1]$ defined by $d \mu=\frac{d \theta}{\ln 2(\theta+1)}$, where $d \theta$ denotes Lebesgue measure. Then $G$ is $\mu$-invariant, i.e., $\mu\left(G^{-1}(E)\right)=\mu(E)$ for every Borel set $E \subseteq[0,1]$ (see [5] for details). From this it follows that the map

$$
V_{G}(f)(\theta)=f(G(\theta)) \quad \text { for } \quad f \in L^{2}(\mu), \theta \in[0,1]
$$

is an isometry. A standard calculation reveals that

$$
V_{G}^{*}(f)(\theta)=\sum_{s=1}^{\infty} f\left(\frac{1}{\theta+s}\right) \frac{1+\theta}{(\theta+s)(\theta+s+1)}
$$

and it is routine to verify that $V_{G}^{*}(f) \in C[0,1]$ when $f \in C[0,1]$. We mention that, symbolically, $V_{G}^{*}$ is the Perron-Frobenius operator of $G$ under $\mu$ and refer the reader to [5, Chapter 2] for details about Perron-Frobenius operators and their connections to continued fractions.

Furthermore, if we embed $C[0,1]$ into $B\left(L^{2}(\mu)\right)$ as $f \mapsto \mathcal{M}_{f}$ where $\mathscr{M}_{f}(g)=f g$, then

$$
V_{G}^{*} \mathscr{M}_{f} V_{G}=\mathscr{M}_{V_{G}^{*}(f)} .
$$

This defines a unital completely positive map, which we will henceforth denote by $\mathrm{G}$, on $C[0,1]$. Then $\mathrm{G}$ is $\mu$-invariant, i.e.,

$$
\int f d \mu=\int \mathrm{G}(f) d \mu \quad \text { for all } \quad f \in C[0,1] .
$$

Furthermore $\mathrm{G}$ acts on the maximal ideals of $C[0,1]$ in the same way that $G$ acts on $[0,1]$. Specifically, for each $\theta \in[0,1]$ define the maximal ideal $J_{\theta}=\{f \in C[0,1]: f(\theta)=0\}$ and for $s \in \mathrm{N}$ let $\theta_{s}=G^{-1}(\theta) \cap\left[\frac{1}{s+1}, \frac{1}{s}\right)$. Then

$$
\mathrm{G}\left(J_{\theta_{s}}\right)=J_{\theta} .
$$

Hence we are looking for an extension of $G$ to $\mathfrak{A}$ that satisfies the natural analogs of (1.2)-(1.4). Therefore, we must first consider what Gauss measure 
should mean on $\mathfrak{X}$. Our first step is proving that every state on $C[0,1]$ has a unique extension to a trace on $\mathfrak{U}$ (Theorem 2.6). D. Mundici showed [7, Theorem 4.5] that the state space of $C[0,1]$ and the space of tracial states on $\mathfrak{U}$ are affinely, weak* homeomorphic. But for our purposes, we will need the extension property from Theorem 2.6.

For reasons that will become clear, we have to slightly modify the natural analogs of (1.2) and (1.3). In particular, we use Theorem 2.6 to define two state extensions, $\phi$ and $\tau$, of $\mu$ and intertwine between these two GNS representations to obtain analogs of (1.2) and (1.3). Let $\left(\pi_{\phi}, L^{2}(\mathfrak{H}, \phi)\right)$ and $\left(\pi_{\tau}, L^{2}(\mathfrak{H}, \tau)\right)$ be the GNS representations of $\mathfrak{U}$ associated with $\phi$ and $\tau$. Since $\phi$ and $\tau$ are extensions of $\mu$, it follows that $L^{2}(\mu) \subseteq L^{2}(\mathfrak{H}, \phi), L^{2}(\mathfrak{H}, \tau)$ and

$$
\left.\pi_{\phi}(f)\right|_{L^{2}(\mu)}=\mathscr{M}_{f} \quad \text { for every } \quad f \in Z(\mathfrak{L}) \cong C[0,1] .
$$

This allows us to prove the main theorem:

THEOREM 1.1. There is a unital completely positive map $\widetilde{\mathrm{G}}: \mathfrak{Y} \rightarrow \mathfrak{A}$ and an isometry $\widetilde{V}_{G}: L^{2}(\mathfrak{H}, \tau) \rightarrow L^{2}(\mathfrak{H}, \phi)$ such that

(1) $\left.\widetilde{\mathrm{G}}\right|_{C[0,1]}=\mathrm{G}$.

(2) $\widetilde{\mathrm{G}}\left(I_{\theta_{s}}\right)=I_{\theta}$, for each $s \in \mathrm{N}$ and $\theta \in[0,1]\left(I_{\theta} \in \operatorname{Max}(\mathfrak{H})\right.$ defined in [1]).

(3) $\left.\widetilde{V}_{G}\right|_{L^{2}(\mu)}=V_{G}$ and $\left.\widetilde{V}_{G}^{*}\right|_{L^{2}(\mu)}=V_{G}^{*}$.

(4) $\widetilde{V}_{G}^{*} \pi_{\phi}(x) \widetilde{V}_{G}=\pi_{\tau}(\widetilde{\mathrm{G}}(x))$ for $x \in \mathfrak{A}$. Hence $\left.\widetilde{V}_{G}^{*} \pi_{\phi}(f) \widetilde{V}_{G}\right|_{L^{2}(\mu)}=\mathscr{M}_{\mathrm{G}(f)}$ for $f \in C[0,1]$.

(5) $\phi(x)=\tau(\widetilde{\mathrm{G}}(x))$ for $x \in \mathfrak{A}$.

For the convenience of the reader, we briefly recall the construction of $\mathfrak{A}$ from [1]. For $n \geq 0$ and $0 \leq k \leq 2^{n}$ we recursively define positive integers $p(n, k)$ and $q(n, k)$ as follows:

$$
\begin{cases}q(n, 0)=q\left(n, 2^{n}\right), \quad p(n, 0)=p\left(n, 2^{n}\right) ; & \\ q(n+1,2 k)=q(n, k), \quad p(n+1,2 k)=p(n, k) & 0 \leq k \leq 2^{n} \\ q(n+1,2 k+1)=q(n, k)+q(n, k+1), & \\ p(n+1,2 k+1)=p(n, k)+p(n, k+1), & 0 \leq k \leq 2^{n} .\end{cases}
$$

Then set $r(n, k)=\frac{p(n, k)}{q(n, k)}$. Then $\mathfrak{A}$ is the inductive limit of the finite dimensional $C^{*}$-algebras

$$
\mathfrak{A}_{n}=\bigoplus_{0 \leq k \leq 2^{n}} M_{q(n, k)} .
$$

with Bratteli diagram as in Figure 1 where the nodes of the $n$th row is labelled with the numbers $r(n, 0), r(n, 1), \ldots, r\left(n, 2^{n}\right)$. 


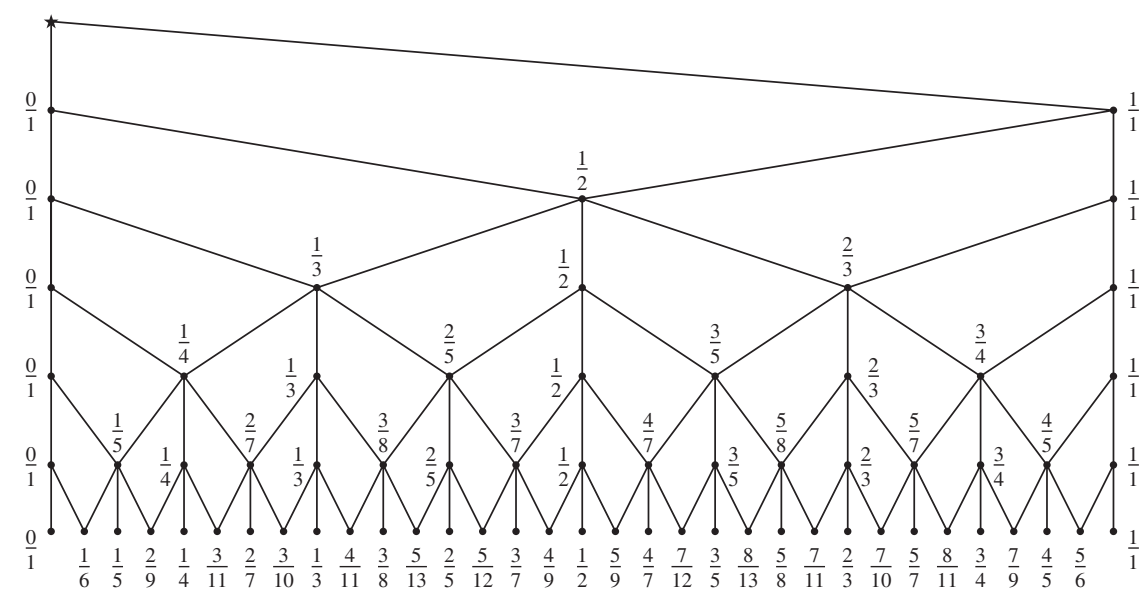

FIGURE 1. Bratteli diagram of $\mathfrak{A}$

For each $n \geq 0$ let $\mathrm{E}_{n}: \mathfrak{A} \rightarrow \mathfrak{A}_{n}$ be conditional expectations such that

$$
\mathrm{E}_{n} \mathrm{E}_{m}=\mathrm{E}_{m} \mathrm{E}_{n} \quad \text { for all } \quad n, m \geq 0 .
$$

The existence of such conditional expectations is guaranteed by Arveson's extension theorem, or since $\mathfrak{A}$ is $\mathrm{AF}$, one can construct such maps explicitly. Furthermore for $0 \leq k \leq 2^{n}$ let $\mathrm{E}_{(n, k)}: \mathfrak{A} \rightarrow M_{q(n, k)} \subseteq \mathfrak{A}_{n}$ be conditional expectations, such that

$$
\mathrm{E}_{(n, k)} \mathrm{E}_{n}=\mathrm{E}_{n} \mathrm{E}_{(n, k)} \quad \text { for } \quad n \geq 0 \text { and } 0 \leq k \leq 2^{n} .
$$

Note that for each $x \in \mathfrak{A}$ we have

$$
\lim _{n \rightarrow \infty} \mathrm{E}_{n}(x)=x .
$$

We will use the following notation throughout: For a unital $C^{*}$-algebra $A$, we let

- Z(A) denote the center of $A$,

- $\mathscr{S}(A)$ denote the state space of $A$,

- $\mathscr{T}(A)$ denote the set of all unital traces of $A$.

- $M_{n}$ denote $n \times n$ matrices over C,

- $\tau_{n}$ the unital trace on $M_{n}$ and $1_{n} \in M_{n}$ the identity.

- For $\frac{p}{q} \in \mathrm{Q} \cap[0,1]$ in reduced form we define

$$
M_{\frac{p}{q}}:=M_{q} \quad \tau_{\frac{p}{q}}:=\tau_{q} \in \mathscr{T}\left(M_{q}\right) .
$$




\section{State Extensions and Conditional Expectation onto $Z(\mathfrak{H})$}

In this section we will construct a conditional expectation from $\mathfrak{A}$ onto $Z(\mathfrak{H})$ that preserves every $\tau \in \mathscr{T}(\mathfrak{H})$. This will provide the key step in the proof of Theorem 2.6.

Definition 2.1. Let $n \geq 0$ and $0 \leq k \leq 2^{n}$. Define $\tau_{(n, k)} \in \mathscr{T}(\mathfrak{H})$ as

$$
\tau_{(n, k)}(x)=\tau_{q(n, k)} \circ \mathrm{E}_{(n, k)}(x) .
$$

The following lemma is immediate from (1.6) and (1.7):

Lemma 2.2. For $n \geq 0,0 \leq k \leq 2^{n}$ and $\ell \geq 0$, we have

$$
\tau_{(n, k)}=\tau_{\left(n+\ell, 2^{\ell} k\right)} .
$$

Proposition 2.3. Let $x \in \mathfrak{A}$. Define the function $f_{x}: \mathrm{Q} \cap[0,1] \rightarrow \mathrm{C}$ as

$$
f_{x}(r(n, k))=\tau_{(n, k)}(x) \quad \text { for } n \geq 0 \text { and } 0 \leq k \leq 2^{n} .
$$

Then $f_{x}$ is well-defined and extends to a continuous function on $[0,1]$.

Proof. If $r(n, k)=r\left(n^{\prime}, k^{\prime}\right)$ with $n^{\prime} \geq n$ then there is an $\ell \geq 0$ such that $n^{\prime}=n+\ell$ and $k^{\prime}=2^{\ell} k$. Hence $f_{x}$ is well-defined by Lemma 2.2.

Let $n \geq 0$ and $0<k<2^{n}$. By the relationships defined in (1.5), the following function is continuous and piecewise affine on $[0,1]$ :

$$
B_{(n, k)}(\theta)= \begin{cases}0 & \text { if } 0 \leq \theta \leq r(n, k-1) \\ q(n, k)(q(n, k-1) \theta & \text { if } r(n, k-1) \leq \theta \leq r(n, k) \\ -p(n, k-1)) & \\ q(n, k)(p(n, k+1) & \text { if } r(n, k) \leq \theta \leq r(n, k+1) \\ -q(n, k+1) \theta) & \text { if } r(n, k+1) \leq \theta \leq 1\end{cases}
$$

We first let $x \in \mathfrak{A}_{n} \subset \mathfrak{A}$ and prove that $f_{x}$ extends to a continuous function on $[0,1]$. Suppose first that $0<2 k+1<2^{n}$ and $\mathrm{E}_{(n, 2 k+1)}(x)=x$. Without loss of generality suppose that $\tau_{(n, 2 k+1)}(x)=1$. We show that $f_{x}=B_{(n, 2 k+1)}$.

It is clear that $\left.f_{x}\right|_{[0, r(n, 2 k)] \cup[r(n, 2 k+2), 1]} \equiv 0$. We now show by induction on $\ell \geq 0$ that

$$
\begin{array}{r}
(\forall \ell \geq 0)\left(\forall 2^{\ell} 2 k<j<2^{\ell}(2 k+2)\right)\left(f_{x}(r(n+\ell, j))\right. \\
\left.=B_{(n, 2 k+1)}(r(n+\ell, j))\right) .
\end{array}
$$


For $\ell=0$, we have $f_{x}(r(n, 2 k+1))=\tau_{(n, 2 k+1)}(x)=1=B_{(n, 2 k+1)}(r(n, 2 k+$ 1)). Suppose now that (2.2) holds for $\ell \geq 0$ and prove (2.2) for $\ell+1$.

If $j=2 i$ is even, then

$$
\begin{aligned}
f_{x}(r(n+\ell+1,2 i)) & =\tau_{(n+\ell+1,2 i)}(x)=\tau_{(n+\ell, i)}(x)=f_{x}(r(n+\ell, i)) \\
& =B_{(n, 2 k+1)}(r(n+\ell, i))=B_{(n, 2 k+1)}(r(n+\ell+1,2 i)) .
\end{aligned}
$$

Now suppose $j=2 i+1$ is odd. Since $B_{(n, 2 k+1)}$ is piecewise affine and due to the relationships in (1.5) we obtain,

$$
\begin{aligned}
B_{(n, 2 k+1)}(r(n+\ell+1,2 i+1)) \\
=\frac{q(n+\ell, i)}{q(n+\ell+1,2 i+1)} B_{(n, 2 k+1)}(r(n+\ell, i)) \\
\quad \quad+\frac{q(n+\ell, i+1)}{q(n+\ell+1,2 i+1)} B_{(n, 2 k+1)}(r(n+\ell, i+1)) \\
=\frac{q(n+\ell, i)}{q(n+\ell+1,2 i+1)} \tau_{(n+\ell, i)}(x)+\frac{q(n+\ell, i+1)}{q(n+\ell+1,2 i+1)} \tau_{(n+\ell, i+1)}(x) \\
=\tau_{(n+\ell+1,2 i+1)}(x)=f_{x}(r(n+\ell+1,2 i+1)) .
\end{aligned}
$$

This shows that (2.2) holds, hence $f_{x}$ extends to a continuous function on $[0,1]$.

Now suppose that $0<2^{m} k<2^{n}$ with $k$ odd and $\mathrm{E}_{\left(n, 2^{m} k\right)}(x)=x$. Then,

$$
x=\mathrm{E}_{(n-m, k)}(x)-\mathrm{E}_{(n-m+1,2 k-1)}(x)-\mathrm{E}_{(n-m+1,2 k+1)}(x) .
$$

So, by the first part of the proof it follows that $f_{x}$ is continuous.

When $x$ is equal to $1 \oplus 0 \oplus \cdots \oplus 0$ or $0 \oplus \cdots \oplus 0 \oplus 1 \in \mathfrak{A}_{n}$, the proof that $f_{x}$ is continuous is exactly the same as above, so we omit the proof. This shows that for every $n \geq 0$ and each $x \in \mathfrak{A}_{n}$ that $f_{x}$ is continuous. Moreover note that the linear map $x \mapsto f_{x}$ defined on $\bigcup_{n=1}^{\infty} \mathfrak{A}_{n}$ is contractive, hence $f_{x}$ is continuous for every $x \in \mathfrak{A}$.

Remark 2.4. We note that the piecewise affine functions $B_{(n, k)}$ appearing in the proof of Proposition 2.3 are precisely those that appear in the proof of [6, Theorem 3.3] computing $K_{0}(\mathfrak{H})$.

As mentioned in the introduction, we have $Z(\mathfrak{H}) \cong C[0,1]$. We now construct an explicit isomorphism. For each $n \geq 0$, define $\mathscr{Z}_{n}: C[0,1] \rightarrow$ $Z\left(\mathfrak{U}_{n}\right) \subset \mathfrak{A}$ by

$$
\mathscr{Z}_{n}(f)=\bigoplus_{0 \leq k \leq 2^{n}} f(r(n, k)) 1_{q(n, k)}
$$


By [1, pg. 977], for each $n \geq 0$ we have $\max \{|r(n, k)-r(n, k+1)|: 0 \leq$ $\left.k<2^{n}\right\}=1 /(n+1)$. Hence for $m \geq n$ we have

$$
\left\|\mathscr{Z}_{n}(f)-\mathscr{Z}_{m}(f)\right\| \leq \sup \left\{\left|f(\theta)-f\left(\theta^{\prime}\right)\right|:\left|\theta-\theta^{\prime}\right| \leq 1 /(n+1)\right\} .
$$

Therefore $\mathscr{Z}_{n}(f)$ is a Cauchy sequence in $\mathfrak{H}$ because $f$ is uniformly continuous on $[0,1]$.

Define $\mathscr{Z}: C[0,1] \rightarrow \mathfrak{U}$ by

$$
\mathscr{Z}(f)=\lim _{n \rightarrow \infty} \mathscr{Z}_{n}(f) .
$$

TheORem 2.5. The map $\mathscr{Z}: C[0,1] \rightarrow Z(\mathfrak{H})$ is a ${ }^{*}$-isomorphism. Moreover the map $\mathrm{E}_{Z}: \mathfrak{U} \rightarrow Z(\mathfrak{U})$ defined by

$$
\mathrm{E}_{Z}(x)=\mathscr{Z}\left(f_{x}\right)
$$

is a conditional expectation such that

$$
\tau\left(\mathrm{E}_{Z}(x)\right)=\tau(x) \quad \text { for every } \quad \tau \in \mathscr{T}(\mathfrak{U}) .
$$

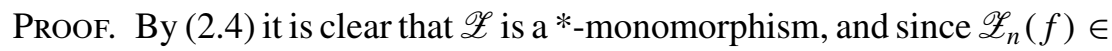
$Z\left(\mathfrak{H}_{n}\right)$ for each $n \geq 0$, it follows that $\mathscr{Z}(f) \in Z(\mathfrak{H})$. We now show that $\mathscr{Z}$ is surjective. Let $n \geq 0$ and $y \in \mathfrak{A}_{n}$. Then

$$
y \in Z\left(\mathfrak{A}_{n}\right) \quad \text { if and only if } \quad y=\bigoplus_{0 \leq k \leq 2^{n}} \tau_{(n, k)}(y) 1_{q(n, k)} .
$$

Let $x \in Z(\mathfrak{R})$. By (2.1) and (2.3) it follows that

$$
\mathscr{Z}_{n}\left(f_{x}\right)=\bigoplus_{0 \leq k \leq 2^{n}} \tau_{(n, k)}(x) 1_{q(n, k)} \in Z\left(\mathfrak{A}_{n}\right) .
$$

Since $x \in Z(\mathfrak{H})$, it follows from (1.8) that

$$
\lim _{n \rightarrow \infty} \operatorname{dist}\left(\mathrm{E}_{n}(x), Z\left(\mathfrak{A}_{n}\right)\right)=0,
$$

from which we deduce by (2.6) that $\mathscr{Z}_{n}\left(f_{x}\right) \rightarrow x$. Therefore

$$
\mathrm{E}_{Z}(x)=\mathscr{Z}\left(f_{x}\right)=\lim _{n \rightarrow \infty} \mathscr{Z}_{n}\left(f_{x}\right)=x .
$$

This shows that $\mathscr{Z}$ is surjective and also that $\mathrm{E}_{Z}$ is a conditional expectation. We now show that $\mathrm{E}_{Z}$ preserves every trace of $\mathfrak{Y}$. Let $\tau \in \mathscr{T}(\mathfrak{H})$. By (1.8) it 
follows that $\tau$ is the weak*-limit of $\tau \circ \mathrm{E}_{n}$. Since $\left.\tau \circ \mathrm{E}_{n}\right|_{\mathfrak{A}_{n}} \in \mathscr{T}\left(\mathfrak{H}_{n}\right)$, there is a convex combination of scalars $\left(\lambda_{(n, k)}\right)_{0 \leq k \leq 2^{n}}$ such that

$$
\tau \circ \mathrm{E}_{n}=\sum_{0 \leq k \leq 2^{n}} \lambda_{(n, k)} \tau_{(n, k)} .
$$

It follows that $\mathscr{T}(\mathfrak{V})$ equals the weak* closure of the convex hull of the set $\left\{\tau_{(n, k)}: n \geq 0,0 \leq k \leq 2^{n}\right\}$. Therefore, we only need to check (2.5) for the traces $\tau_{(n, k)}$. To this end, let $x \in \mathfrak{A}$ then

$$
\begin{aligned}
\tau_{(n, k)}(x) & =f_{x}(r(n, k))=\tau_{(n, k)}\left(\mathscr{Z}_{n}\left(f_{x}\right)\right) \\
& =\tau_{(n, k)}\left(\mathscr{Z}\left(f_{x}\right)\right)=\tau_{(n, k)}\left(\mathrm{E}_{Z}(x)\right) .
\end{aligned}
$$

THEOREM 2.6. The restriction map $\left.\tau \mapsto \tau\right|_{Z(\mathfrak{I})}$ defines a weak* homeomorphism from $\mathscr{T}(\mathfrak{H})$ onto $\mathscr{S}(C[0,1])$. In particular, every state on $Z(\mathfrak{H})$ has a unique tracial extension to $\mathfrak{A}$.

Proof. Injectivity and weak*-continuity of the inverse both follow from (2.5). By (2.8) it follows that the restriction of $\tau_{(n, k)}$ to $Z(\mathfrak{Q}) \cong C[0,1]$ is the Dirac measure $\delta_{\{r(n, k)\}}$, which shows surjectivity.

\section{Ideals of $\mathfrak{A}$ and traces of $\mathfrak{A}$}

Definition 3.1. Fix $\theta \in[0,1]$. Define $\tau_{\theta}^{\mathfrak{H}} \in \mathscr{T}(\mathfrak{H})$ as the unique tracial extension of the Dirac measure $\delta_{\{\theta\}} \in \mathscr{S}(C[0,1])$ given by Theorem 2.6.

For each $\theta \in[0,1]$, we recall the maximal ideals $I_{\theta} \subset \mathfrak{U}$ defined in [1, Proposition 4]. The following is a consequence of the proof of [1, Proposition 4] and the correspondence made in Theorem 2.6.

Corollary 3.2. Fix $\theta \in[0,1]$. Then

$$
I_{\theta}=\left\{x \in \mathfrak{U}: \tau_{\theta}^{\mathfrak{A}}\left(x^{*} x\right)=0\right\} .
$$

Fix $\frac{p(n, k)}{q(n, k)}=\frac{p}{q} \in \mathrm{Q} \cap(0,1)$ in reduced form. We define the *-homomorphism

$$
\pi_{\frac{p}{q}}: \mathfrak{U} \rightarrow M_{\frac{p}{q}}
$$

as "evaluation along the path $r(n, k), r(n+1,2 k), \ldots, r\left(n+\ell, 2^{\ell} k\right), \ldots$ in the Bratteli diagram." Specifically, we have

$$
\pi_{\frac{p}{q}}(x)=\lim _{\ell \rightarrow \infty} \mathrm{E}_{\left(n+\ell, 2^{\ell} k\right)}(x) \quad \text { for } \quad x \in \mathfrak{A} .
$$


It then follows from [1, Proposition 4.(ii)] that, $\operatorname{ker}\left(\pi \frac{p}{q}\right)=I_{\frac{p}{q}}$. We note that

$$
\tau_{\frac{p}{q}}^{\mathfrak{P}}(x)=\tau_{\frac{p}{q}}\left(\pi_{\frac{p}{q}}(x)\right) \quad \text { for every } \quad x \in \mathfrak{A} .
$$

\section{Construction of $\tilde{G}$}

In this section we construct our noncommutative Gauss map $\widetilde{G}: \mathfrak{A} \rightarrow \mathfrak{A}$. Let $s \geq 1$. As in $[1,(3.1)]$ we define

$$
\mathscr{J}_{s}:=\mathscr{J}\left(\left[\frac{1}{s+1}, \frac{1}{s}\right]\right)=\bigcap_{\theta \in\left[\frac{1}{s+1}, \frac{1}{s}\right]} I_{\theta} .
$$

By Theorem 2.6 and Section 3 we have

$$
\mathscr{J}_{s}=\operatorname{ker}\left(\bigoplus_{\frac{1}{s+1}<\frac{p}{q} \in \mathrm{Q}<\frac{1}{s}} \pi_{\frac{p}{q}}\right) .
$$

For each $s \geq 1$ the Bratteli diagram of $\mathfrak{U} / \mathscr{J}_{s}$ is the subdiagram of the Bratteli diagram of $\mathfrak{U}$ obtained by deleting all of the nodes

$$
\{r(n, k): r(n, k) \notin[1 /(s+1), 1 / s]\} \cup\{r(n, k): n<s\},
$$

and deleting all edges connected to any of these nodes. See Figure 2 for the Bratteli diagram of $\mathfrak{U} / \mathscr{J}_{2}$.

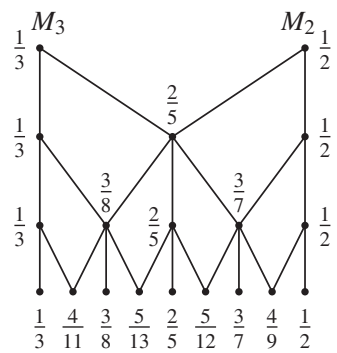

FIGURE 2. Bratteli diagram of $\mathfrak{H} / \mathscr{J}_{2}$

For each $s \geq 1$, define the homeomorphism $g_{s}:[0,1] \rightarrow[1 /(s+1), 1 / s]$ as

$$
g_{s}(\theta)=\frac{1}{\theta+s},
$$

and recall that these maps are the building blocks for the commutative Gauss map $\mathrm{G}: C[0,1] \rightarrow C[0,1]$ defined in (1.1). Then consider the induced isomorphism

$$
\left(g_{s}\right)_{*}: C[1 /(s+1), 1 / s] \rightarrow C[0,1] \quad \text { defined by } \quad\left(g_{s}\right)_{*}(f)=f \circ g_{s} .
$$


Since our goal is to extend $\mathrm{G}$ to a map on $\mathfrak{A}$, we first consider extensions of the maps $\left(g_{s}\right)_{*}$ as maps from $\mathfrak{U} / \mathscr{J}_{s}$ into $\mathfrak{A}$. Unfortunately, there is no hope for these extensions to also be isomorphisms. Indeed, by considering the Bratteli diagrams of $\mathfrak{U}$ and $\mathfrak{H} / \mathscr{J}_{s}$ it is clear that $K_{0}(\mathfrak{H}) \cong K_{0}\left(\mathfrak{H} / \mathscr{J}_{s}\right)$, but there is no unit-preserving, positive homomorphism that implements this isomorphism. Hence $\mathfrak{A} \not \mathfrak{A} / \mathscr{J}_{s}$.

We do the next best thing by defining a (non-unital) *-monomorphism $H_{s}: \mathfrak{A} \rightarrow \mathfrak{H} / \mathscr{J}_{s}$ and a unital completely positive (UCP for short) map $G_{s}: \mathfrak{U} / \mathscr{J}_{s} \rightarrow \mathfrak{U}$ such that $G_{s} H_{s}=\mathrm{id}_{\mathfrak{I}}$, and such that $G_{s}$ is an extension of $\left(g_{s}\right)_{*}$. More importantly, the maps $G_{s}$ and $H_{s}$ will provide a nice relationship (see (4.9)) between $\mathscr{T}(\mathfrak{H})$ and $\mathscr{T}\left(\mathfrak{H} / \mathscr{J}_{s}\right)$.

Before constructing our maps, we pause to outline our strategy for extending the maps $\left(g_{s}\right)_{*}$.

We consider the case $s=1$. The map $g_{1}$ shrinks $[0,1]$ in half and then flips it. We want to mimic this action on the Bratteli diagrams of $\mathfrak{A}$ and $\mathfrak{H} / \mathscr{J}_{1}$ (see Figure 3). For example, $g_{1}\left(\frac{1}{2}\right)=\frac{2}{3}$, so we would like to construct a unital, completely positive map $T: M_{2} \rightarrow M_{3}$ such that induced map $\widetilde{T}$ : $L^{2}\left(M_{2}, \tau_{2}\right) \rightarrow L^{2}\left(M_{3}, \tau_{3}\right)$ is an isometry. But any map $T$ that satisfies these conditions is necessarily a unital *-homomorphism; an impossibility.

This is essentially the reason why we must consider another non-tracial state extension $\phi$ of $\mu$ in Theorem 1.1(5). Consider the state $\omega \in \mathscr{S}\left(M_{3}\right)$ defined by

$$
\omega(x)=\tau_{3}\left(\left[\begin{array}{ccc}
\frac{3}{2} & 0 & 0 \\
0 & \frac{3}{2} & 0 \\
0 & 0 & 0
\end{array}\right] x\right)
$$

and the *-homomorphism $T: M_{2} \rightarrow M_{3}$ defined by

$$
T(x)=\left[\begin{array}{ll}
x & \\
& 0
\end{array}\right]
$$

Let $S: M_{3} \rightarrow M_{2}$ be the cutdown map such that $S T=i d_{M_{2}}$.

Let $\widetilde{T}$ denote the induced map from $L^{2}\left(M_{2}, \tau_{2}\right)$ into $L^{2}\left(M_{3}, \omega\right)$. Then $\widetilde{T}$ is an isometry and

$$
\widetilde{T}^{*} \pi_{\omega}(x) \widetilde{T}=\pi_{\tau_{2}}(S x) \quad \text { for } \quad x \in M_{3} .
$$

So, our plan is to construct maps on $\mathfrak{A}$ and $\mathfrak{H} / \mathscr{J}_{s}$ built up from maps similar to the maps $T$ and $S$, that are as close to trace preserving as possible, and then to take an inductive limit.

We now turn to the construction. For $n \geq 0$, let $A_{n} \in M_{2^{n+1}+1,2^{n}+1}\left(\mathrm{Z}^{+}\right)$be the transition matrices for the connecting homomorphisms from $\mathfrak{A}_{n}$ into $\mathfrak{A}_{n+1}$ 
such that

$$
\mathfrak{U}=\underline{\lim }\left(\mathfrak{U}_{n}, A_{n}\right) .
$$

For example we have,

$$
A_{0}=\left[\begin{array}{ll}
1 & 0 \\
1 & 1 \\
0 & 1
\end{array}\right] \in M_{3,2}, \quad A_{1}=\left[\begin{array}{lll}
1 & 0 & 0 \\
1 & 1 & 0 \\
0 & 1 & 0 \\
0 & 1 & 1 \\
0 & 0 & 1
\end{array}\right] \in M_{5,3}, \quad \ldots
$$

In an effort to keep notation to a minimum the matrices $A_{n}$ will stand for both the transition matrices for the connecting homomorphisms and for the homomorphisms themselves. For $n \geq 0$, we define

$$
\left(\mathfrak{U} / \mathscr{J}_{s}\right)_{n}:=\bigoplus_{0 \leq k \leq 2^{n}} M_{\frac{q(n, k)}{p(n, k)+s q(n, k)}}=\bigoplus_{0 \leq k \leq 2^{n}} M_{g_{s}(r(n, k))}
$$

By the description of the Bratteli diagram of $\mathfrak{U} / \mathscr{J}_{s}$ (see also Figure 2) given above it follows that

$$
\mathfrak{U} / \mathscr{J}_{s}=\underset{\lim }{\longrightarrow}\left(\left(\mathfrak{U} / \mathscr{J}_{s}\right)_{n}, A_{n}\right)
$$

Let $\ell^{\infty}(s)$ denote the $s$ dimensional, commutative $C^{*}$-algebra. Consider the $C^{*}$-algebra,

$$
\ell^{\infty}(s) \otimes \mathfrak{U}=\lim _{\longrightarrow}\left(\ell^{\infty}(s) \otimes \mathfrak{A}_{n}, \mathrm{id}_{\ell^{\infty}(s)} \otimes A_{n}\right)
$$

Define $S=\left[\begin{array}{llll}1 & 1 & \ldots & 1\end{array}\right] \in M_{1, s}$. It is easy to see (using only the fact that $\left.A_{n} \in M_{2^{n+1}+1,2^{n}+1}\left(\mathbf{Z}^{+}\right)\right)$that

$$
A_{n}\left(S \otimes 1_{2^{n}+1}\right)=\left(S \otimes 1_{2^{n+1}+1}\right) 1_{s} \otimes A_{n} \quad \text { for every } n \geq 0 .
$$

Hence, for each $n \geq 0$ we are able to define a $*$-homomorphism

$$
\sigma_{n}: \ell^{\infty}(s) \otimes \mathfrak{U}_{n}=\bigoplus_{0 \leq k \leq 2^{n}} \ell^{\infty}(s) \otimes M_{\frac{p(n, k)}{q(n, k)}} \rightarrow \bigoplus_{0 \leq k \leq 2^{n}} M_{\frac{q(n, k)}{p(n, k)+s q(n, k)}}=\left(\mathfrak{H} / \mathscr{J}_{s}\right)_{n}
$$

given by the transition matrix $S \otimes 1_{2^{n}+1} \in M_{2^{n}+1, s\left(2^{n}+1\right)}$ such that the following diagram commutes for every $n \geq 0$ :

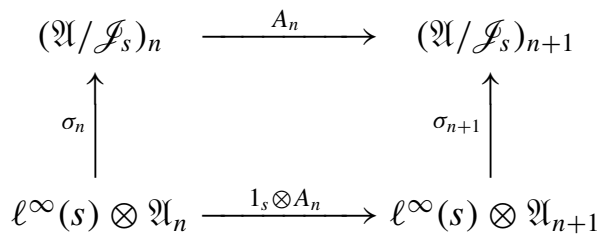


Let $e_{1}, \ldots, e_{s} \in \ell^{\infty}(s)$ denote the standard basis. For each $n \geq 0$ define the UCP map $V_{n}:(\mathfrak{H} / \mathscr{J})_{n} \rightarrow \ell^{\infty}(s) \otimes \mathfrak{A}_{n}$ by

$$
V_{n}(x)=\sigma_{n}^{-1}\left(\sum_{i=1}^{s} \sigma_{n}\left(e_{i} \otimes 1_{\mathfrak{F}_{n}}\right) x \sigma_{n}\left(e_{i} \otimes 1_{\mathfrak{P}_{n}}\right)\right)
$$

Define $\psi_{s} \in \mathscr{S}\left(\ell^{\infty}(s)\right)$ by

$$
\psi_{s}\left(\sum_{i=1}^{s} \alpha_{i} e_{i}\right)=\frac{1}{s} \sum_{i=1}^{s} \alpha_{i} .
$$

It now follows from (4.4) that the following diagram commutes for all $n \geq 0$ :
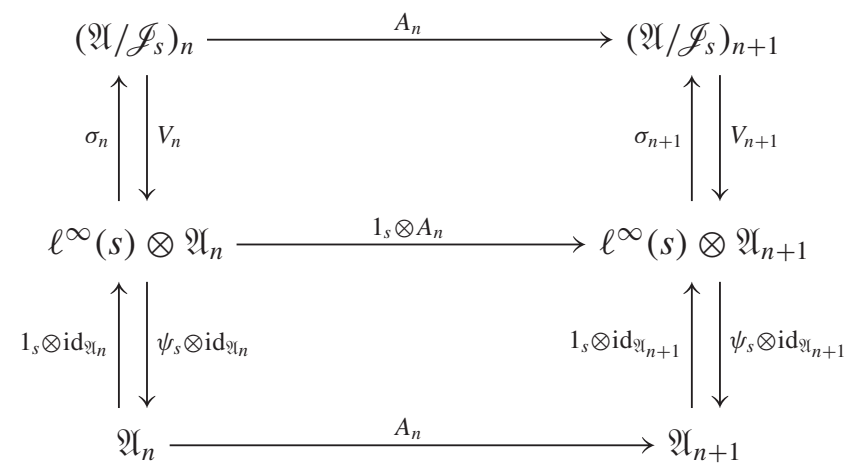

Furthermore,

(4.6) $\quad\left(\psi_{s} \otimes \operatorname{id}_{\mathscr{V}_{n}}\right) \circ V_{n} \circ \sigma_{n} \circ\left(1_{s} \otimes \mathrm{id}_{\mathscr{F}_{n}}\right)=\operatorname{id}_{\mathscr{A}_{n}} \quad$ for all $\quad n \geq 0$.

Now, let $x \in M_{\frac{p(n, k)}{q(n, k)}} \subset \mathfrak{A}_{n}$ and $y \in M_{\frac{q(n, k)}{p(n, k)+s q(n, k)}} \subset\left(\mathfrak{U} / \mathscr{J}_{s}\right)_{n}$. Set $p=p(n, k)$ and $q=q(n, k)$. Then, by basic properties of the trace it follows that (4.7)

$$
\begin{aligned}
& \tau_{\frac{q}{p+s q}}\left(\sigma_{n}\left(1_{s} \otimes x\right) y\right) \\
& =\tau_{\frac{q}{p+s q}}\left(\sigma_{n}\left(1_{s} \otimes x\right) \sum_{i=1}^{s} \sigma_{n}\left(e_{i} \otimes 1_{\mathfrak{U}_{n}}\right) y \sigma_{n}\left(e_{i} \otimes 1_{\mathfrak{A}_{n}}\right)\right) \\
& =\frac{s q}{p+s q} \psi_{s} \otimes \tau_{\frac{p}{q}}\left(\sigma_{n}^{-1}\left(\sigma_{n}\left(1_{s} \otimes x\right) \sum_{i=1}^{s} \sigma_{n}\left(e_{i} \otimes 1_{\mathfrak{I}_{n}}\right) y \sigma_{n}\left(e_{i} \otimes 1_{\mathfrak{A}_{n}}\right)\right)\right) \\
& =\frac{s q}{p+s q} \psi_{s} \otimes \tau_{\frac{p}{q}}\left(\left(1_{s} \otimes x\right) V_{n}(y)\right) \\
& =\frac{s q}{p+s q} \tau_{\frac{p}{q}}\left(x\left(\psi_{s} \otimes \operatorname{id}_{\mathfrak{I}_{n}}\left(V_{n}(y)\right)\right) .\right.
\end{aligned}
$$


We now let $\widetilde{V}_{s}: \mathfrak{H} / \mathscr{J}_{s} \rightarrow \mathfrak{U}$ be the inductive limit of the maps $\left(\psi_{s} \otimes \mathrm{id}_{\mathfrak{H}_{n}}\right) \circ V_{n}$, which is well-defined by (4.5). We also let $\widetilde{\sigma}_{s}: \mathfrak{U} \rightarrow \mathfrak{U} / \mathscr{J}_{s}$ be the inductive limit of the maps $\sigma_{n} \circ\left(1_{s} \otimes \mathrm{id}_{\mathscr{U}_{n}}\right)$, which again are well-defined by (4.5).

Figure 3 displays the mapping $\widetilde{\sigma}_{1}$ in terms of the Bratteli diagrams of $\mathfrak{A}$ and $\mathfrak{U} / \mathscr{J}_{1}$.

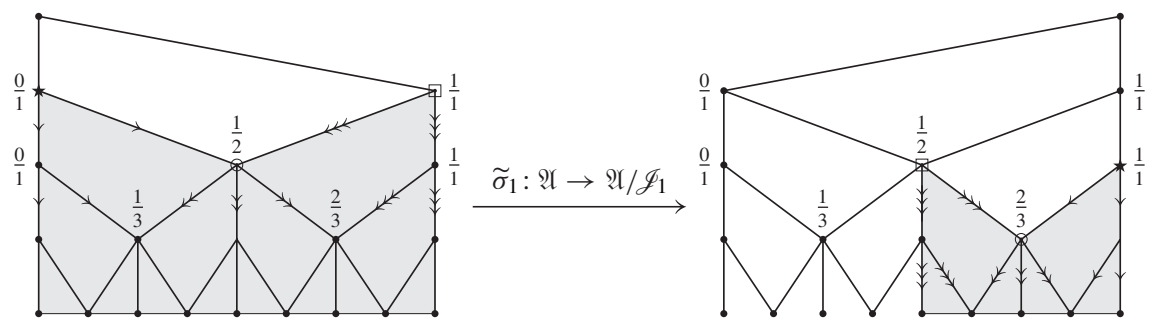

FIGURE 3. The map $\widetilde{\sigma}_{1}$

Set $\pi=\bigoplus_{\frac{1}{s+1}<\frac{p}{q}<\frac{1}{s}} \pi_{\frac{p}{q}}$ and identify $\mathfrak{U} / \mathscr{J}_{s}$ with $\pi(\mathfrak{V})$ by (4.1). By the ChoiEffros lifting theorem in [3], there is a UCP lifting $\Phi: \mathfrak{H} / \mathscr{J}_{s} \rightarrow \mathfrak{U}$ of $\pi$. Then let

$$
G_{s}:=\widetilde{V}_{s} \circ \pi: \mathfrak{A} \rightarrow \mathfrak{A} \quad \text { and } \quad H_{s}:=\Phi \circ \widetilde{\sigma}_{s}: \mathfrak{A} \rightarrow \mathfrak{A} .
$$

By (4.6), it follows that

$$
G_{s} H_{s}=\mathrm{id}_{\mathfrak{U}} .
$$

It is also routine to verify, using the definitions of $\sigma_{n}$ and $V_{n}$, that

$$
G_{s}\left(x H_{s}(y)\right)=G_{s}(x) y \quad \text { for every } \quad x, y \in \mathfrak{U} .
$$

By (4.7), we have the following relationship for every $x, y \in \mathfrak{A}$, and $\frac{p}{q} \in$ $\mathrm{Q} \cap[0,1]:$

$$
\begin{aligned}
\tau_{g_{s}(p / q)}^{\mathscr{A}}\left(H_{s}(x) y\right) & =\tau_{\frac{q}{p+s q}}^{\mathfrak{Q}}\left(H_{s}(x) y\right)=\tau_{\frac{q}{p+s q}}\left(\pi_{\frac{q}{p+s q}}\left(H_{s}(x) y\right)\right) \\
& =\frac{s q}{p+s q} \tau_{\frac{p}{q}}\left(\pi_{\frac{p}{q}}\left(x G_{s}(y)\right)\right)=\frac{s q}{p+s q} \tau_{\frac{p}{q}}^{\mathfrak{Q}}\left(x G_{s}(y)\right) \\
& =s g_{s}(p / q) \tau_{\frac{p}{q}}^{\mathfrak{H}}\left(x G_{s}(y)\right) .
\end{aligned}
$$

Therefore, by Theorem 2.6, for any $\theta \in[0,1]$ we have

$$
\tau_{g_{s}(\theta)}^{\mathfrak{Q}}\left(H_{s}(x) y\right)=s g_{s}(\theta) \tau_{\theta}^{\mathfrak{A}}\left(x G_{s}(y)\right) .
$$

Therefore, by Corollary 3.2, it follows that for any $\theta \in[0,1]$, we have

$$
G_{s}\left(I_{g_{s}(\theta)}\right)=I_{\theta} .
$$


Moreover, by the description of $Z(\mathfrak{Q})$ given in Theorem 2.5 and (4.9) it is clear that

$$
G_{s}(f)=f \circ g_{s} \quad \text { for every } f \in C[0,1] .
$$

For each $s \geq 1$, define $f_{s} \in Z(\mathfrak{H}) \cong C[0,1]$ as

$$
f_{s}(\theta)=\frac{\theta+1}{(\theta+s)(\theta+s+1)}
$$

Let us now define $\widetilde{\mathrm{G}}: \mathfrak{A} \rightarrow \mathfrak{A}$ as

$$
\widetilde{\mathrm{G}}(x)=\sum_{s=1}^{\infty} G_{s}(x) f_{s} .
$$

\section{Proof of Theorem 1.1}

In this section we will prove the five assertions from Theorem 1.1. First note that Theorem 1.1(1) follows from (1.1) and (4.11), and (2) follows from (4.10).

First define $\phi_{0}:=\tau_{0}^{\mathfrak{A}}$. Then, let $\theta \in[0,1]$ with $\frac{1}{s+1}<\theta \leq \frac{1}{s}$ for some $s \geq 1$. Then define

$$
\phi_{\theta}(x)=\tau_{\theta}^{\mathfrak{A}}\left(H_{s}(1)\right)^{-1} \tau_{\theta}^{\mathfrak{A}}\left(H_{s}(1) x\right)=\frac{1}{s \theta} \tau_{\theta}^{\mathfrak{A}}\left(H_{s}(1) x\right) \quad \text { for every } \quad x \in \mathfrak{A} .
$$

Recall that Gauss measure $\mu$ on $[0,1]$ is defined as the probability measure $d \mu=\frac{d \theta}{\ln 2(\theta+1)}$, where $d \theta$ is Lebesgue measure. Let $\phi \in \mathscr{S}(\mathfrak{U})$ be the direct integral of the states $\phi_{\theta}$ over $\mu$, i.e.,

$$
\phi(x)=\int_{0}^{1} \phi_{\theta}(x) d \mu(\theta) .
$$

Let $\tau \in \mathscr{T}(\mathfrak{Q})$ be the unique tracial extension of $\mu$ provided by Theorem 2.6. By uniqueness we have

$$
\tau=\int_{0}^{1} \tau_{\theta}^{\mathfrak{A}} d \mu(\theta) .
$$

Notice that for every $f \in C[0,1]$ and $x \in \mathfrak{A}$, we have

$$
\phi_{\theta}(f x)=f(\theta) \phi_{\theta}(x) \quad \text { and } \quad \tau_{\theta}^{\mathfrak{A}}(f x)=f(\theta) \tau_{\theta}^{\mathfrak{Y}}(x)
$$

It also follows from (5.1) that $\phi$ restricted to $C[0,1]$ is Gauss measure $\mu$.

For any state $\psi \in \mathscr{S}(\mathfrak{U})$, let $\left(L^{2}(\mathfrak{H}, \psi), \pi_{\psi}\right)$ denote the GNS representation of $\psi$ and $\langle\cdot, \cdot\rangle_{\psi}$ the inner product on $L^{2}(\mathfrak{A}, \psi)$. For $x \in \mathfrak{A}$, we will denote 
by $x_{\psi}$ the image of $x$ in $L^{2}(\mathfrak{H}, \psi)$ and denote by $\mathfrak{A}_{\psi}$ the dense subspace of $L^{2}(\mathfrak{A}, \psi)$ consisting of the $x_{\psi}$.

By the definitions of $\phi$ and $\tau$, we can decompose

$$
L^{2}(\mathfrak{A}, \phi)=\int_{0}^{1} L^{2}\left(\mathfrak{H}, \phi_{\theta}\right) d \mu(\theta) \quad \text { and } \quad L^{2}(\mathfrak{A}, \tau)=\int_{0}^{1} L^{2}\left(\mathfrak{A}, \tau_{\theta}^{\mathfrak{A}}\right) d \mu(\theta)
$$

Furthermore, by (5.1) we have

$$
L^{2}([0,1], \mu) \subset L^{2}(\mathfrak{N}, \phi) \quad \text { and } \quad L^{2}([0,1], \mu) \subset L^{2}(\mathfrak{A}, \tau)
$$

via the identifications

$$
f=\int_{0}^{1} f(\theta) 1_{\phi_{\theta}} d \mu(\theta) \quad \text { and } \quad f=\int_{0}^{1} f(\theta) 1_{\tau_{\theta}^{\vartheta}} d \mu(\theta)
$$

for $f \in L^{2}(\mu)$.

We now define an isometry $\widetilde{V}_{G}: L^{2}(\mathfrak{A}, \tau) \rightarrow L^{2}(\mathfrak{A}, \phi)$ that satisfies (3)(5) in Theorem 1.1. As short hand notation, for each vector $\eta \in L^{2}(\mathfrak{A}, \phi)$ and Borel set $E \subset[0,1]$ we will write

$$
\eta 1_{E}:=\int_{E} \eta(\theta) d \mu(\theta) \in \int_{0}^{1} L^{2}\left(\mathfrak{H}, \phi_{\theta}\right) d \mu(\theta)
$$

For each $s \geq 1$, define operators on $\mathfrak{H}_{\phi}$ and $\mathfrak{H}_{\tau}$ respectively as

$$
\widetilde{H}_{s}\left(x_{\tau}\right)=H_{s}(x)_{\phi} 1_{\left[\frac{1}{s}, \frac{1}{s+1}\right]} \text { and } \quad \widetilde{G}_{s}\left(x_{\phi}\right)=\left(G_{s}(x) f_{s}\right)_{\tau} .
$$

Clearly these maps are contractive, so they extend to operators on $L^{2}(\mathfrak{H}, \phi)$ and $L^{2}(\mathfrak{A}, \tau)$ respectively. Now define

$$
\widetilde{V}_{G}=W O T-\sum_{s=1}^{\infty} \widetilde{H}_{s}
$$

We now show that $\widetilde{V}_{G}$ is an isometry. Let us first recall $f_{s}$ from (4.12) and note that

$$
\sum_{s=1}^{\infty} f_{s}(\theta)=1 \quad \text { for every } \quad \theta \in[0,1]
$$

We will implicitly use this fact throughout the rest of the proof of Theorem 1.1. 
Let $d u$ denote Lebesgue measure. Then

$$
\begin{aligned}
\left\langle\widetilde{V}_{G}\left(x_{\tau}\right), \widetilde{V}_{G}\left(x_{\tau}\right)\right\rangle_{\phi} & \\
& =\sum_{s=1}^{\infty} \int_{\frac{1}{s+1}}^{\frac{1}{s}} \phi_{\theta}\left(H_{s}(x)^{*} H_{s}(x)\right) d \mu(\theta) \\
& =\sum_{s=1}^{\infty} \int_{\frac{1}{s+1}}^{\frac{1}{s}} \frac{1}{s \theta} \tau_{\theta}^{\mathfrak{Q}}\left(H_{s}\left(x^{*} x\right)\right) d \mu(\theta) \\
& \left.=\sum_{s=1}^{\infty} \int_{\frac{1}{s+1}}^{\frac{1}{s}} \tau_{\frac{1}{\theta}-s}^{\mathfrak{A}}\left(x^{*} x\right) d \mu(\theta) \quad \text { by }(4.9)\right) \\
& =\frac{1}{\ln 2} \sum_{s=1}^{\infty} \int_{0}^{1} \tau_{u}^{\mathfrak{H}}\left(x^{*} x\right) \frac{u+1}{(u+s)(u+s+1)} \frac{d u}{u+1} \quad\left(\text { with } u=\frac{1}{\theta}-s\right) \\
& =\int_{0}^{1} \tau_{u}^{\mathfrak{H}}\left(x^{*} x\right)\left(\sum_{s=1}^{\infty} f_{s}(u)\right) d \mu(u) \\
& =\left\langle x_{\tau}, x_{\tau}\right\rangle_{\tau} .
\end{aligned}
$$

We now calculate $\widetilde{V}_{G}^{*}$. Let $x, y \in \mathfrak{A}$, then

$$
\begin{aligned}
\left\langle\widetilde{V}_{G}\left(x_{\tau}\right), y_{\phi}\right\rangle_{\phi} & =\sum_{s=1}^{\infty} \int_{\frac{1}{s+1}}^{\frac{1}{s}} \phi_{\theta}\left(y^{*} H_{s}(x)\right) d \mu(\theta) \\
& =\sum_{s=1}^{\infty} \int_{\frac{1}{s+1}}^{\frac{1}{s}} \frac{1}{s \theta} \tau_{\theta}^{\mathfrak{A}}\left(y^{*} H_{s}(x)\right) d \mu(\theta) \\
& =\sum_{s=1}^{\infty} \int_{\frac{1}{s+1}}^{\frac{1}{s}} \tau_{\frac{1}{\theta}-s}^{\mathfrak{A}}\left(G_{s}(y)^{*} x\right) d \mu(\theta) \quad(\text { by }(4.9)) \\
& =\sum_{s=1}^{\infty} \int_{0}^{1} \tau_{\theta}^{\mathfrak{A}}\left(G_{s}(y)^{*} x\right) f_{s}(\theta) d \mu(\theta) \quad \text { (Reasoning as in (5.4)) } \\
& =\sum_{s=1}^{\infty} \int_{0}^{1} \tau_{\theta}^{\mathfrak{A}}\left(G_{s}(y)^{*} f_{s} x\right) d \mu(\theta) \quad \text { (By (5.1)) } \\
& =\left\langle x_{\tau}, \sum_{s=1}^{\infty} \widetilde{G}_{s}\left(y_{\phi}\right)\right\rangle_{\tau} . \\
& =\left\langle x_{\tau}, \widetilde{\mathrm{G}}(y)_{\tau}\right\rangle_{\tau} .
\end{aligned}
$$

We now show (3). By (5.2) let $f=\int_{0}^{1} f(\theta) 1_{\tau_{\theta}} d \mu(\theta) \in L^{2}(\mu) \subset L^{2}(\mathfrak{H}, \tau)$. 
Then

$$
\begin{aligned}
\widetilde{V}_{G}(f) & =\sum_{s=1}^{\infty} \int_{\frac{1}{s+1}}^{\frac{1}{s}} f\left(\frac{1}{\theta}-s\right)\left(H_{s}(1)\right)_{\phi_{\theta}} d \mu(\theta) \\
& =\sum_{s=1}^{\infty} \int_{\frac{1}{s+1}}^{\frac{1}{s}} f\left(\frac{1}{\theta}-s\right) 1_{\phi_{\theta}} d \mu(\theta) \\
& =f \circ G \in L^{2}(\mathfrak{A}, \phi) .
\end{aligned}
$$

Similarly, one shows that $\left.\widetilde{V}_{G}^{*}\right|_{L_{2}(\mu)}=V_{G}^{*}$. This proves (3).

We now show (4). It follows from the definition of $G_{s}$ that for every $s \geq 1$ we have

$$
\int_{\left[\frac{1}{s+1}, \frac{1}{s}\right]^{c}} L^{2}\left(\mathfrak{A}, \phi_{\theta}\right) d \mu(\theta) \subset \operatorname{ker}\left(\widetilde{G}_{s}\right)
$$

From this and (4.8) it follows that for every $x, y \in \mathfrak{A}$ we have

$$
\begin{aligned}
\tilde{V}_{G}^{*} \pi_{\phi}(x) \tilde{V}_{G}\left(y_{\tau}\right) & =\widetilde{V}_{G}^{*}\left(\sum_{s=1}^{\infty}\left(x H_{s}(y)\right)_{\phi} 1_{\left[\frac{1}{s+1}, \frac{1}{s}\right]}\right) \\
& =\sum_{s=1}^{\infty}\left(G_{s}\left(x H_{s}(y)\right) f_{s}\right)_{\tau} \\
& =\sum_{s=1}^{\infty}\left(G_{s}(x) y f_{s}\right)_{\tau} \quad(\mathrm{By}(4.8)) \\
& =\pi_{\tau}(\mathrm{G}(x)) y_{\tau} .
\end{aligned}
$$

By (5.5) we have $\widetilde{V}_{G}\left(1_{\tau}\right)=1_{\phi}$, from which it follows that

$$
\phi(x)=\left\langle x_{\phi}, 1_{\phi}\right\rangle=\left\langle x_{\phi}, \tilde{V}_{G}\left(1_{\tau}\right)\right\rangle=\left\langle\mathrm{G}(x)_{\tau}, 1_{\tau}\right\rangle=\tau(\mathrm{G}(x)) .
$$

This proves (5) and finishes the proof of Theorem 1.1.

Acknowledgement. Many thanks are due to Florin Boca for suggesting this problem to me, several helpful conversations, and for providing code for the figures in this article.

\section{REFERENCES}

1. Boca,Florin P., An AF algebra associated with the Farey tessellation, Canad. J. Math. 60 (2008), 975-1000.

2. Bratteli, Ola, The center of approximately finite-dimensional $C^{*}$-algebras, J. Functional Analysis 21(1976), 195-202. 
3. Choi, Man Duen, and Effros, Edward G., The completely positive lifting problem for $C^{*}$ algebras, Ann. of Math. (2) 104 (1976), 585-609.

4. Effros, Edward G., and Shen, Chao Liang, Approximately finite $C^{*}$-algebras and continued fractions, Indiana Univ. Math. J. 29 (1980), 191-204.

5. Iosifescu, Marius, and Kraaikamp, Cor, Metrical theory of continued fractions, Math. and its Applications 547, Kluwer, Dordrecht 2002.

6. Mundici, Daniele, Farey stellar subdivisions, ultrasimplicial groups, and $K_{0}$ of $A F C^{*}$ algebras, Adv. in Math. 68 (1988), 23-39.

7. Mundici, Daniele, Revisiting the Farey AF algebra, preprint, 2008.

8. Pedersen, Gert K., $C^{*}$-algebras and their automorphism groups, London Math. Soc. Monographs 14, Academic Press, London 1979.

UNIVERSITÉ DE FRANCHE-COMTÉ

16 ROUTE DE GRAY

BESANÇON 25030

FRANCE

E-mail: ceckhard@gmail.com 